\title{
Two Cancers Arising from Different Mesenchymal Origins: An Unexpected Connection over Time and Space
}

\author{
Natascha Putri', Sze Min Lek, Nicholas Brian Shannon', Joey Wee-Shan Tan1, \\ Grace Hwei Ching Tan ${ }^{1}$, Claramae Shulyn Chia ${ }^{1}$, Melissa Ching Ching Teo ${ }^{1}$, Choon Hua Thng', \\ Timothy Kwang Yong Tay ${ }^{3}$, Chin-Ann Johnny Ong ${ }^{1 *}$, Tina Puay Theng Koh ${ }^{{ }^{*}}$
}

\begin{abstract}
${ }^{1}$ Division of Surgical Oncology, National Cancer Centre Singapore, Singapore City, Singapore ${ }^{2}$ Division of Oncologic Imaging, National Cancer Centre Singapore, Singapore City, Singapore ${ }^{3}$ Department of Anatomical Pathology, Singapore General Hospital, Singapore City, Singapore Email: ^tina.koh.p.t@singhealth.com.sg, *johnny.ong.c.a@singhealth.com.sg
\end{abstract}

How to cite this paper: Putri, N., Lek, S.M., Shannon, N.B., Tan, J.W.-S., Tan, G.H.C., Chia, C.S., Teo, M.C.C., Thng, C.H., Tay, T.K.Y., Ong, C.-A.J. and Koh, T.P.T. (2019) Two Cancers Arising from Different Mesenchymal Origins: An Unexpected Connection over Time and Space. Case Reports in Clinical Medicine, 8, 99-104.

https://doi.org/10.4236/crcm.2019.85012

Received: April 12, 2019

Accepted: May 11, 2019

Published: May 14, 2019

Copyright $\odot 2019$ by author(s) and Scientific Research Publishing Inc. This work is licensed under the Creative Commons Attribution International License (CC BY 4.0).

http://creativecommons.org/licenses/by/4.0/

\section{(c) (†) Open Access}

\begin{abstract}
Sarcomas are rare tumours, accounting for $1 \%$ of all malignancies. They are histologically diverse, presenting with more than 50 different subtypes. Sarcoma can be broadly divided into two categories-soft tissue sarcoma and bone sarcoma. Extraskeletal osteosarcoma is a unique subtype which is histologically similar to bone osteosarcoma but resides within soft tissue and has no attachments to bone. We present a rare case of a patient who initially presented with an extraskeletal osteosarcoma that was complicated by a chronic chest wall sinus and lymphedema of the left arm. He later developed enlarging left chest wall nodules at the same region that were proven to be dedifferentiated liposarcoma on biopsy 20 years later. We examine the occurrence of both extremely rare diseases of differing pathologies over the same site but separated by an extraordinarily long interval, and possible new associations to account for this phenomenon.
\end{abstract}

\section{Keywords}

Sarcoma, Extraskeletal Osteosarcoma, Dedifferentiated Liposarcoma, Osteogenic Sarcoma

\section{Introduction}

Sarcomas are rare and uncommon tumours, representing $1 \%$ of adult malignancies [1]. They are a heterogeneous group of tumours of mesenchymal origin that can occur anywhere in the body. Sarcomas are broadly divided into two catego- 
ries-soft tissue sarcoma and bone sarcoma, of which liposarcoma and osteosarcoma are the most common histological subtypes respectively.

Extraskeletal osteosarcoma is a unique subtype of sarcoma, presenting with similar histology to that of osteosarcoma but lies within soft tissue and is not attached to any bones [2]. It represents approximately $1 \%-2 \%$ of all soft tissue sarcomas, and behaves aggressively, carrying mortality rates up to $80 \%$ [3]. Extraskeletal osteosarcomas are different from its primary counterpart of osseous origin-it occurs rarely in the younger population, and are poorly responsive to chemotherapy, with local and distant failures in more than $80 \%$ of those affected. Disease-free intervals have been found to range from two months to ten years [4]. Specifically, primary thoracic extraskeletal osteosarcoma is even rarer, and only 60 cases have been reported to date with the most common presentation coming from the lungs. The prognosis is usually extremely bleak, as the majority of patients reported had demised within months due to rapid disease progression, and overall survival at 1 year stands only at $41.8 \%$ and decreases to $22.3 \%$ at 5 years [5].

Herein, we report an interesting case of a patient who underwent resection for extraskeletal osteosarcoma 20 years ago and recently presented with a dedifferentiated liposarcoma at the same site.

\section{Case Summary}

Our patient is an 81-year-old Chinese male with comorbidities of hypertension and hyperlipidemia and a significant smoking history. He previously received treatment in another hospital for a left chest wall extraskeletal osteosarcoma for which he underwent wide excision and reconstruction with mesh and latissimus dorsi flap in 1997. He did not receive further adjuvant treatment. He remained well and disease-free until 2016 when he noted a persistent chronic chest wall sinus as well as left upper limb lymphedema that had failed conservative management. Computed tomography scan of his chest showed a $2.7 \mathrm{~cm}$ soft tissue thickening over the left anterior chest wall surgical site with no evidence of pulmonary metastasis or vascular obstruction. Clinical examination revealed no visible or palpable nodules, and incisional biopsy of the chest wall sinus proved to be inflamed granulation tissue with no evidence of malignancy or any infective organisms. He declined further investigations for possible disease recurrence then and only consented to treatment for the left upper limb lymphedema. He underwent left upper limb lymphovascular anastomosis in 2017 with good results. A repeat computed tomography scan of his chest done in 2018 showed interval stability of the soft tissue density, as well as oedematous changes present over the left anterior and lateral chest wall muscles. There were no overt masses detected.

However, in early 2019, his symptoms recurred with worsening lymphedema of the left upper limb associated with left chest wall nodularity inferior to the previous reconstruction site that was suspicious of underlying recurrence. $\mathrm{He}$ was otherwise well with no other systemic symptoms. On examination, our pa- 
tient had a few clusters of non-tender, hard nodules on the left lateral chest wall at the angle of the ribs just below the nipple with no surrounding erythema or ulcers (Figure 1(a) and Figure 1(b)). A long well healed scar extending from the anterior chest wall to the scapular region is seen, the left clavicle was previously surgically resected and the infraclavicular fossa is depressed relative to the right. There is marked winging of the scapula but the patient retained a good range of movement of his left upper limb. There was no cervical or axillary lymphadenopathy bilaterally. Of note, there is marked lymphedema of the left upper limb. At the midsternal region, there is a small chronic chest wall sinus (Figure 1(c)).

A magnetic resonance imaging scan of the chest was performed which showed enlarging clusters of enhancing soft tissue nodules located at the region of the previous left latissimus dorsi flap repair in the intermuscular fascial plane (Figure 2).

There was progressive increase in size of the left chest wall nodularity, but no intraosseous abnormality or gross destruction of the bones. He underwent ultrasound-guided biopsy of the left chest wall mass, and histopathology revealed the lesions to be dedifferentiated liposarcoma, which was MDM2 positive (Figure 3). This case was discussed at the multi-disciplinary tumour board meeting and curative surgical resection proposed. However, the patient declined surgery in view of his current relatively well state and elected to continue monitoring during his last review three weeks ago. He will be due for follow-up in a month.

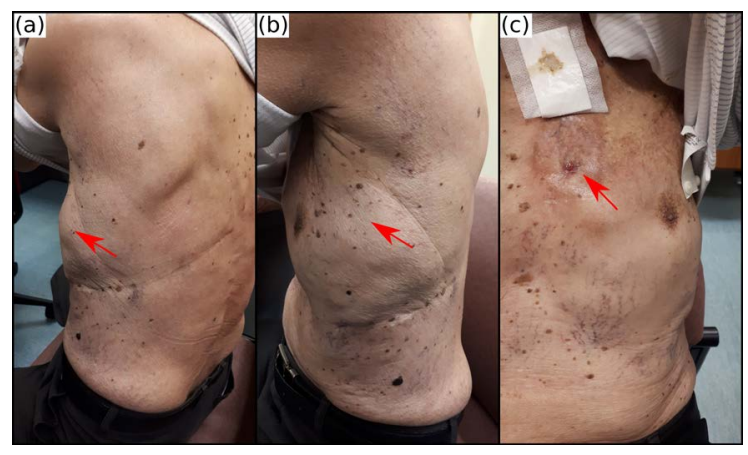

Figure 1. ((a) and (b)) Photograph of lateral chest wall of patient demonstrating presence of nodules with no surrounding erythema or ulcers at the site of latissimus dorsi flap; (c) Photograph of chronic discharging sinus at mid sternal region.

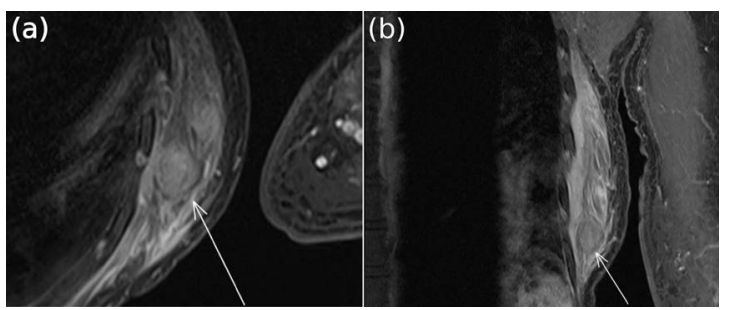

Figure 2. Magnetic resonance imaging scan of (a) transverse plane and (b) coronal plane showing cluster of enhancing soft tissue nodules in the region of the left latissimus dorsi flap. 


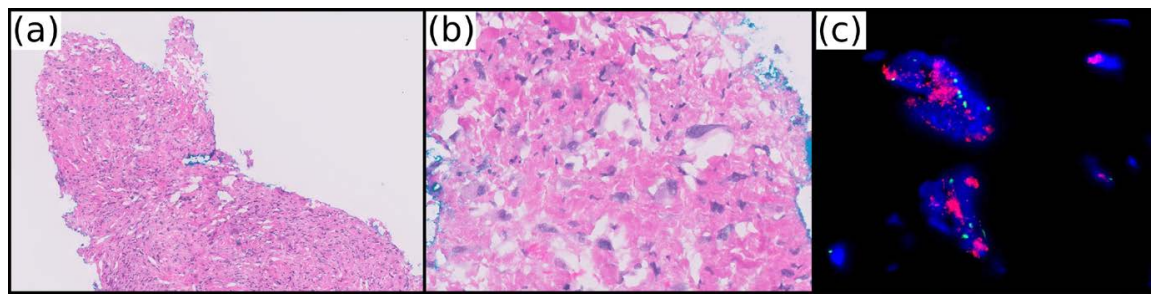

Figure 3. (a) Low magnification view showing a moderately cellular population of atypical spindle cells within a fibrotic stroma devoid of lipogenic differentiation (H \& E 10X); (b) Higher magnification view of the spindle cells showing nuclear atypia with occasional marked nuclear pleomorphism (H \& E 40×); (c) Cells hybridized with dual colour MDM2 DNA probe labelled with Spectrum GreenTM fluorophores. Amplification of the MDM2 gene is represented as multiple red signals.

\section{Discussion}

Among soft tissue sarcomas, liposarcoma is the most common and accounts for approximately $20 \%$ of all cases [6]. These tumours affect adults with a peak incidence at around 60 years of age. About $10 \%$ progress to dedifferentiated liposarcomas and is most commonly non-lipogenic and pleomorphic. Heterologous differentiation can occur in $10 \%$ of dedifferentiated liposarcomas and present with myogenic, osteo- or chondrosarcomatous or angiosarcomatous components.

Extraskeletal osteosarcoma is a rare entity that accounts for $1 \%-2 \%$ of all soft tissue sarcomas and $6 \%$ of all osteosarcomas. They too occur typically in the elderly with a predilection to the extremities and retroperitoneum. It is a malignant mesenchymal tumour that directly produces neoplastic osteoid or bone and diagnosis requires a careful exclusion of heterologous osteosarcomatous differentiation arising in a variety of malignant neoplasms.

This is an interesting case whereby the surprise diagnosis of dedifferentiated liposarcoma in this current instance raises several unique and rare possibilities; whether the current presentation is a recurrence linked to the previous history of extraskeletal osteosarcoma, or a de novo occurrence of a second primary malignancy arising from cells of a different mesenchymal origin. Another possibility could be a misdiagnosis in the index presentation, where for instance, the initial tumour could have been a dedifferentiated liposarcoma all along, but only the predominant osteosarcomatous component was reported.

To our knowledge, there are no published anecdotes of two different primaries of these unrelated mesenchymal cell types, arising in the same patient at the same site spaced twenty years apart, which makes this novel case noteworthy. One predisposing factor which could have led to the development of a secondary malignancy is the chronic inflammation from the persistent sinus tract or upper limb lymphedema. However, what is reported in literature so far have been lymphangiosarcomas such as in the case of Stewart-Treves syndrome [7], and no association with dedifferentiated liposarcoma has been demonstrated before.

Alternatively, if this new tumour is a recurrence arising from his initial disease, the extremely long disease-free interval and the disparate mesenchymal 
cells of origin would make this case highly unusual. Osteogenic sarcomas have very poor prognosis with 5-year overall survival rates being reported as low as $11.7 \%$ [2] [8] [9] [10]. The established natural history of dedifferentiated liposarcomas, where $90 \%$ arise de novo while only $10 \%$ occur in recurrence, also run contrary to this current presentation [11] [12]. There have been no documented cases in existing literature describing this condition to date.

Another unlikely explanation calls into question the initial diagnosis of extraskeletal osteosarcoma established 20 years ago. The use of molecular markers to distinguish the various sarcoma subtypes has started to become available only recently, such as MDM2 and CDK4 positivity being pathognomonic of liposarcoma. In the absence of MDM2 testing and histopathological review being performed on the first tumour specimen obtained years ago, this opens the possibility of the original diagnosis being a dedifferentiated liposarcoma with osteogenic components being erroneously identified as the primary malignancy. Although uncommon, there have been sporadic case reports describing dedifferentiated liposarcomas with osteosarcomatous components or dedifferentiation to osteosarcoma [13] [14]. In this hypothetical situation, it is also remarkable for dedifferentiated liposarcoma to remain dormant and only recur two decades later, as the known mean time to recurrence is often within two years [1].

\section{Conclusion}

This case emphasizes the need for continued vigilance in the surveillance of sarcoma patients as the disease may have an unprecedentedly long disease-free interval. More importantly, it also exposes the gaps in our current understanding about the behavior of osteosarcomas and dedifferentiated liposarcoma as the temporal sequence of this presentation runs contrary to their established natural history. More can be done to expand the role of molecular markers to distinguish the various subtypes so as to identify the exact mesenchymal cell of origin for these rare tumours and determine the optimal treatment required.

\section{Conflicts of Interest}

The patient provided written informed consent for the publication of this case report and its accompanying images.

\section{References}

[1] Knebel, C., et al. (2017) Prognostic Factors and Outcome of Liposarcoma Patients: A Retrospective Evaluation over 15 Years. BMC Cancer, 17, 410. (In English)

[2] Wang, H., et al. (2018) Extraskeletal Osteosarcoma: A Large Series Treated at a Single Institution. Rare Tumors, 10, 1-7.

[3] McCarter, M.D., Lewis, J.J., Antonescu, C.R. and Brennan, M.F. (2000) Extraskeletal Osteosarcoma: Analysis of Outcome of a Rare Neoplasm. Sarcoma, 4, 119-23. (In English) https://doi.org/10.1080/13577140020008084

[4] Hoch, M., Ali, S., Agrawal, S., Wang, C. and Khurana, J.S. (2013) Extraskeletal Osteosarcoma: A Case Report and Review of the Literature. Journal of Radiology Case 
Reports, 7, 15-23. (In English) https://doi.org/10.3941/jrcr.v7i7.1245

[5] Qian, J., Zhang, X.-Y., Gu, P., Shao, J.-C., Han, B.-H. and Wang, H.-M. (2017) Primary Thoracic Extraskeletal Osteosarcoma: A Case Report and Literature Review. Journal of Thoracic Disease, 9, E1088-E1095. https://doi.org/10.21037/jtd.2017.11.111

[6] Dalal, K.M., Kattan, M.W., Antonescu, C.R., Brennan, M.F. and Singer, S. (2006) Subtype Specific Prognostic Nomogram for Patients with Primary Liposarcoma of the Retroperitoneum, Extremity, or Trunk. Annals of Surgery, 244, 381-391. (In English)

[7] Mesli, S.N., Ghouali, A.K., Benamara, F., Taleb, F.A., Tahraoui, H. and Abi-Ayad, C. (2017) Stewart-Treves Syndrome Involving Chronic Lymphedema after Mastectomy of Breast Cancer. Case Reports in Surgery, 2017, Article ID: 4056459. (In English) https://doi.org/10.1155/2017/4056459

[8] Nystrom, L.M., Reimer, N.B., Reith, J.D., Scarborough, M.T. and Gibbs Jr., C.P. (2016) The Treatment and Outcomes of Extraskeletal Osteosarcoma: Institutional Experience and Review of The Literature. Iowa Orthopedic Journal, 36, 98-103. (In English)

[9] Sio, T.T., et al. (2016) Extraskeletal Osteosarcoma: An International Rare Cancer Network Study. American Journal of Clinical Oncology, 39, 32-36.

[10] Thampi, S., Matthay, K.K., Boscardin, W.J., Goldsby, R. and DuBois, S.G. (2014) Clinical Features and Outcomes Differ between Skeletal and Extraskeletal Osteosarcoma. Sarcoma, 2014, Article ID: 902620. https://doi.org/10.1155/2014/902620

[11] Fujii, T., et al. (2013) Retroperitoneal Dedifferentiated Liposarcoma with Osteosarcomatous Components: A Case Report. International Journal of Clinical and Experimental Pathology, 6, 1427-31. (In English)

[12] Bui, B., et al. (2011) Advanced Well-Differentiated/Dedifferentiated Liposarcomas: role of Chemotherapy and Survival. Annals of Oncology, 23, 1601-1607.

[13] Sun, W.B., Sun, X.Y. and Cao, D.D. (2012) Dedifferentiated Liposarcoma of the Retroperitoneum with Osteosarcomatous Component. Clinics and Practice, 2, e33. (In English)https://doi.org/10.4081/cp.2012.e33

[14] Yu, L., Jung, S., Hojnowski, L. and Damron, T. (2005) Dedifferentiated Liposarcoma of Soft Tissue with High-Grade Osteosarcomatous Dedifferentiation. RadioGraphics, 25, 1082-1086. https://doi.org/10.1148/rg.254045204 